\title{
Fake Information Detection Techniques
}

\author{
Henil Chopada, Maitri Patel, Rushikesh Desai, Divya Ebenezer Nathaniel
}

\begin{abstract}
Fake or unverified information spreads same as actual facts on the internet, hence maybe going viral and influencing the general public opinion and their choices. fake news represents the maximum present-day forms of fake and unverified facts, respectively, and need to be detected as soon as possible for averting their results. The interest in efficient detection techniques has been therefore growing very rapid in the remaining years. In this paper we present survey on the specific techniques to computerized detection of fake news proposed in the latest literature. Particularly, this paper focus on five main aspects. First, we record and discuss the various definitions of fake news that have been considered in various literatures. Second, we highlight how the collection of applicable data for simulation of fake information detection is tough and we present the numerous approaches, which have been adopted to accumulate this information, additionally the publicly available datasets. Third, we describe the features that have been used in various fake news detection techniques. Fourth, we provide an evaluation of various techniques used for detection. In the end, we discuss future directions that could be considered for this problem.
\end{abstract}

Keywords: data mining, text mining, machine learning, deep learning, natural language processing, classification, fake news

\section{INTRODUCTION}

$T_{\text {he role of traditional information channels such as }}$ newspapers and TV on how we collect and take news has become less salient than in the past. surely, the growth of social media platforms has a highly aided role in this shift. As mentioned in Zubiaga et al. [29], social media have turned a critical publishing tool for journalists and the preeminent intake method for citizens looking for the latest news. Journalists may use social media to record on public opinions about breaking news stories, and even to look potential new stories, whereas citizens may follow the development of breaking news and events through official channels or through posts of their own network (e.g. friends, family, public figures) and social networks have proved to be highly useful during crisis situations, as they are capable to spread breaking news much faster than old media.

However, this definite impact of the social media comes at a price: the absence of administration and fact-checking over posts makes social media a prolific ground for the spread of unverified and/or untrue information [29]. People often publish posts or share other people's posts confirming

Revised Manuscript Received on May 20, 2020.

Henil Chopada, Student, Department of Computer Science and Engineering, Babaria Institute of Technology, Vadodara, Gujarat, India

Maitri Patel, Student, Department of Computer Science and Engineering, Babaria Institute of Technology, Vadodara, Gujarat, India.

Rushikesh Desai, Student, Department of Computer Science and Engineering, Babaria Institute of Technology, Vadodara, Gujarat, India.

Mrs. Divya Ebenezer Nathaniel, Assistant Professor, Department of Computer Science and Engineering, Babaria Institute of Technology, Vadodara, Gujarat, India. neither the origin nor the information validness and reliableness. Oftentimes, it is enough for a news article with attractive headline to be shared thousands of times, even if it contains possibly false content. Fake news is not, however, a product of the digital communication age. Before the arrival of the internet, journalists were tasked with substantiation and fact-checking of news and sources [12], thus making the picture of public opinion to fake news more limited. Nowadays, social media provides a fertile ground for the spread of unverified information among a larger number of users, thus resulting deep influence on the public insight and the opinion of any event.

Another issue is the problem of rumors, in the context of social media. An unverified assertion having potential to spread over the whole network and is made by users on social media platforms is defined as rumor. The claim can might out to be either true or false, with the end choice being a sure problem for the community. Similar to fake news, the spread of false rumors can cause severe damage even in a short period.

Here, we focus on five main aspects: definitions of various fake information, data collection for their detection purpose, feature extraction and selection from the collected dataset, different techniques for analysis and detection, and future directions. The paper is organized as follows. In Section III we categorize fake news and define them. In Section IV, we differentiate features which can be extracted. Section V introduces and discusses the different techniques used in recent work in this field. In Section VI we discuss possible future research directions. Section VII we recapitulate the paper.

\section{FALSE INFORMATION BASICS}

\section{A. Fake news}

"Fake news" after the 2016 U.S. Presidential Campaign, has come to be the in-practice expression for identifying false information in mainstream media. However, research on fake news usually uses a more restrictive definition. Fake news is "a news article that is purposely and verifiably false". This definition considers two key aspects: purposeless and verifiability. Fake news is, therefore, news articles that are purposely written to misguide or misinform readers but can be verified as false by means of other sources.

In [12] a categorization of fake news has been introduced. In particular, the authors focus on serious fabrications, large scale hoaxes, and humourous fakes. Serious fabrications are the prototypic form of fake news, i.e. articles with a malicious intention, that often go viral through social media. Large scale hoaxes are reports of fraudulent information covered up as proper news [12]. 
Fake Information Detection Techniques

Usually, such hoaxes are on a larger scale as compared to simple news article and often targeting public figures or ideas. Finally, humorous fakes are scripted in order to amuse readers, who are considered to be aware of the comical intent of the author. Examples are satiric pieces masqueraded as

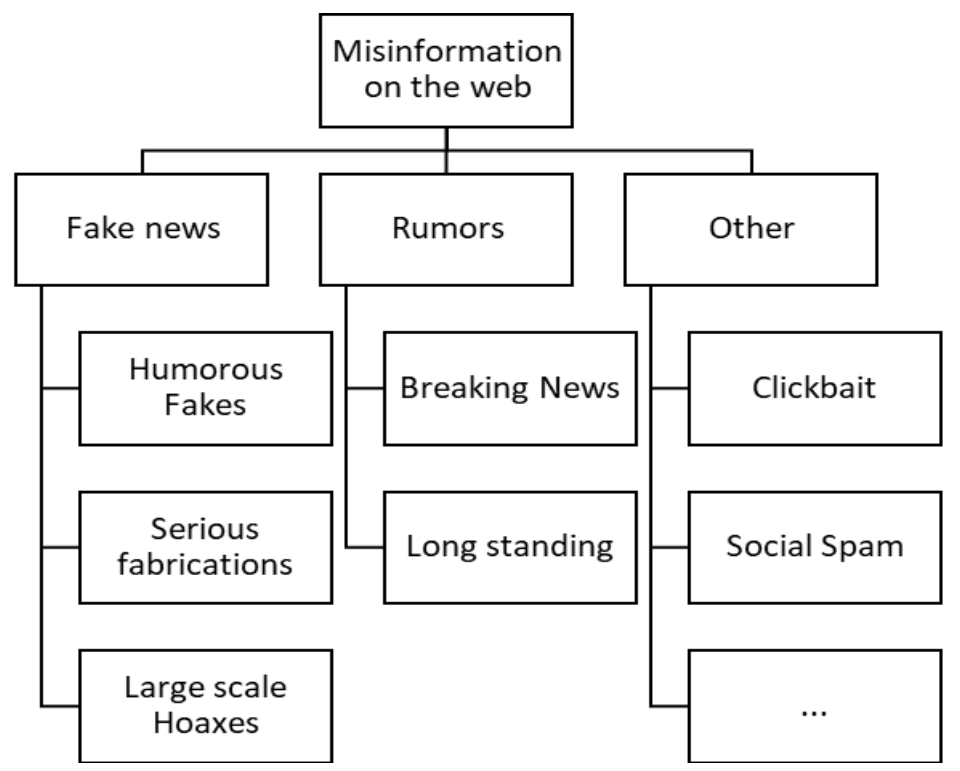

Fig 1. Categorization of fake news

real news, such as the ones produced by websites like The Onion and lercio.it.

\section{B. Rumors}

To offer a formal definition of the rumor is not straightforward. In reality, researchers have mentioned distinctive interpretations. One of the most extensively followed definitions comes from the authors in [2]. Of their information statements in circulation" are defined as rumors. moreover, [14] defines a rumor more particularly as a "circulating tale of questionable veracity, which is seemingly credible but difficult to confirm, and produces enough skepticism and/or anxiety". For this definition, a rumor has to produce an impactful response on its audience. But, we can argue that those definitions stick to the "unverified" feature of the information. This unverified information could be authentic, partly authentic, completely fake or stay unverified [29]. Other works have defined rumors as circulating false information. In this case, the unverified component of records is disregarded, and the primary awareness is posed on its veracity. techniques and scope of such research continue to be however just like the ones adopting the previously presented definition.

Ultimately, different research has attempted to categories rumors with respect to their type, scope, and characteristics. for instance, From a more realistic standpoint, Zubiaga et al. [29] have apparently split rumors into fundamental classes. On one side, long-standing rumors are information that circulates for long durations of time and may in no way be proven as genuine or fake. While, breaking news rumors are maximum not unusual, and appear in connection with breaking information stories. They can be the product of accidental incorrect information, but could also show to be deceptive in nature. The researcher is highly interested in breaking news because of the truth that such rumors can prove to be more dangerous in a short period of time, and need to be identified as soon as possible so as to keep away from their spread, in particular, if their cause is malicious. research, "unverified and instrumentally applicable

\section{DATA COLLECTION}

While collecting relevant data for analysis on fake news and rumors on the web researchers have to face a sequent of problems. First, as we discussed in Section II, the analyst has to manage different types of fake information in the context of web and social media platforms. Second, the amount of false information is a small portion of online content produced every day, even if we limit our focus on news articles and posts discussing breaking news. Third, social media companies have today rigorous policies for what concerns the analysis of data produced by their users. At last, given different misinformations, several different tasks have been proposed in the literature. For each task, researchers may need to apply different means of collecting and annotation of data. For these reasons, a few benchmark datasets are publicly available today.

In this section, we will mention few publicly available datasets and present an overview of data that are relevant to analyze the phenomena of fake information on web.

\section{A. Fake news}

Malicious websites are generally source of Fake news articles, specifically created to spread misinformation. They are repeatedly shared later on social media platforms by authors, malicious users working with them or internet bots, as well as inattentive users who do not bother to check the source of the article ahead sharing it.

In fact, the most straightaway method aimed at deciding a potential fake news article is to evaluate its source. Certain fake news websites are built to resemble qualified news outlets, by mimicking both the visual prospect and the domain name. For example, ABCnews.com.co looks similar to $\mathrm{ABCnews.com}$. This is done in order to delude users and make them believe that they are browsing and sharing from a good source of information. 
Other sources of fake news can be websites promoting conspiracy theories, alternate facts, and alternative medicine. For this reason, such websites can be used to harvest articles, which have a high chance of being false. However, it could be misleading if we just infer the veracity of a fraction of news solely based on its source. Moreover, sometimes verified source could also spread fake news. This could happen for example by error, or for the hurry of publishing breaking news without decently checking sources ahead. Thus, a proper annotation of data conducted by experts on particular affair and with access to many different sources is necessary.

Authors in [24] presents existing approaches in the context of knowledge-based fake news modeling and recognition. Human experts-based Expert-oriented fact-checking evaluate the veracity of the news using expert's knowledge about the event. This technique is used by websites such as Snopes ${ }^{1}$ and FactCheck.org ${ }^{2}$. But, issue with export-oriented fact-checking is time and resources required for the detection. A possible substitute for fact checking is to use crowdsourcing, by this we could exploit the "wiseness of the crowds", this idea is implemented in services such as Fiskkit ${ }^{3}$ . Finally, Shu et al. [24] mentions computational-oriented fact-checking models, which are based on algorithms and outside resources like knowledge graphs to assess both their veracity and check worthy pieces of news. In particular, to efficiently annotate datasets of fake news we can use crowdsourcing-oriented and expert-oriented fact-checking.

In addition, we could build reliable collection of fake news using the abovementioned fact-checking services. Finally, humorous and satirical websites, the well-known example is The Onion ${ }^{4}$, an American satirical website. The authors' intention, in this case, is to amuse readers not to mislead.

\section{B. Rumors}

Rumors are mostly studied on social media. The literature on how datasets ought to be amassed for rumors detection and evaluation in most cases focuses on two principal techniques, specifically top-down and bottom-up collection techniques [29]. the top-down strategy calls for some form of a-priori expertise about target rumors. in particular, rumors are generally accrued, after they spread on social media, by searching for a specific set of key phrases and tags that describe the rumor. The proposed method is pretty straightforward to put in force and for that reason has been employed in numerous researches related to rumor detection and verification [3] . In this context rumor debunking web sites are often used to extract reliable keywords for retrieving posts about those rumors on social media [17] and as a source to discover the most interesting rumors.

Fundamental downsides of top-down collection strategies are i) rumors should be recognized a-priori, making such datasets no longer suitable for the discovery of breaking news sort of rumors, and ii) retrieving all social media posts related to a given rumor is difficult due to difficulty on social media API offerings.

On the opposite, a bottom-up method is especially geared toward collecting potential rumors in breaking news. This collection approach has been proposed in [16,21]. the principle idea is to gather as many social media posts as possible during a certain time window. Then, the accrued posts are evaluated through human annotators as a timeline of activities in an effort to annotate them with numerous levels of evaluation (e.g. rumor/on rumor, true/false, and many others.). This method has some of advantages over a top-down approach. But it poses some trouble that must be held into account. specifically, it is really costlier in terms of both human resources and computational resources. Ultimately, it may also appear that in the collection stage a confined number of rumors of interest emerge [29].

\section{Publicly available datasets}

Given the above-mentioned difficulties in assembling relevant data, not many datasets have been composed and even fewer are publicly available.

Fake News. To the best of our knowledge, an widely accepted benchmark dataset for fake news detection has not been produced yet [24]. The primary reasons for this are the problems of collecting relevant data and difficulty in providing a clear definition of fake news, and the. However, some publicly available resources deserve to be mentioned. Constructing a dataset containing statements for instance, made by politicians or public figures labeled with their veracity, from social media is targeted by many researchers. LIAR [26] contains short political statements, obtained through the website Poli- tiFact.com. Each statement is annotated with the writer, the circumstance, a veracity tag and a justification for such tag. A dataset with both news articles and rumored claims, annotated with their veracity has been provided in [15] by Ferreira and Vlachos.

The authors in [18] have focused on Facebook users. Starting from BuzzFeed ${ }^{5}$, the composed dataset contains webpage links from posts produced by nine verified Facebook users (6 hyperpartisan publishers and 3 mainstream publishers). Each post is annotated and fact-verified for veracity by BuzzFeed journalists. The interesting view of this dataset is that it considers fake news from a more social media-oriented position. CREDBANK [11] is a large-scale dataset, containing 60 million tweets. Tweets are sorted into events by means of topic modeling techniques. Each matter is annotated for credibility via Mechanical Turk. Although a credibility judgment cannot be straightaway associated with a veracity score, it may be used as a good estimation for veracity classification. From a non-research point of view, it is interesting to cite $B S$ Detector $^{6}$, developed by Kaggle ${ }^{7}$. It is a web-crawler with knowledge about fake news websites, that has been used to create a dataset by monitoring such websites. But this dataset cannot be considered as a standard dataset.

\section{FEATURE EXTRACTION}

In this section, we describe the information that are taken in to consideration for the analysis and also discuss different approaches to extract it. Distinguished between content-based and context-based approaches for feature extraction when we are just focusing in fake news detection. 
Fake Information Detection Techniques

\section{A. Content features}

Content features are usually directly available from texts. The advantage of using such features is that given the right tools for the analysis, textual features are easily available. Basic knowledge from the text like Sentences, Tokens, Lemmas, Part-of-Speech (PoS) and structured representation can be obtained using NLP toolkits such as Stanford CoreNLP ${ }^{5}$ and NLTK ${ }^{6}$, which gives more favorable for further analysis.

Syntactic features, such as frequency of specific part-of-speech patterns, the number of nouns, verbs and adjective, have been exploited, for example, in [19]. In addition, sentence complexness has been identified as an indicator for the reliability of the information as well as index of subjectiveness.

Lexical features concern actual word usage in texts. In particular, the most straightaway approach is to exploit the most striking content words or expressions (e.g. bi-grams and tri-grams) as features $[\underline{18,20}]$. Finally, [18] has used features based on novelty of words found in social media posts.

Semantic features are often obtained using advanced NLP approaches. For example, opinion mining and sentiment analysis extract features based on the emotions and opinions presented in the text [2]. Word embeddings []ㅡ, a distributional semantics technique, have been successfully used for fake news and rumors detection, especially in deep learning and machine learning models.

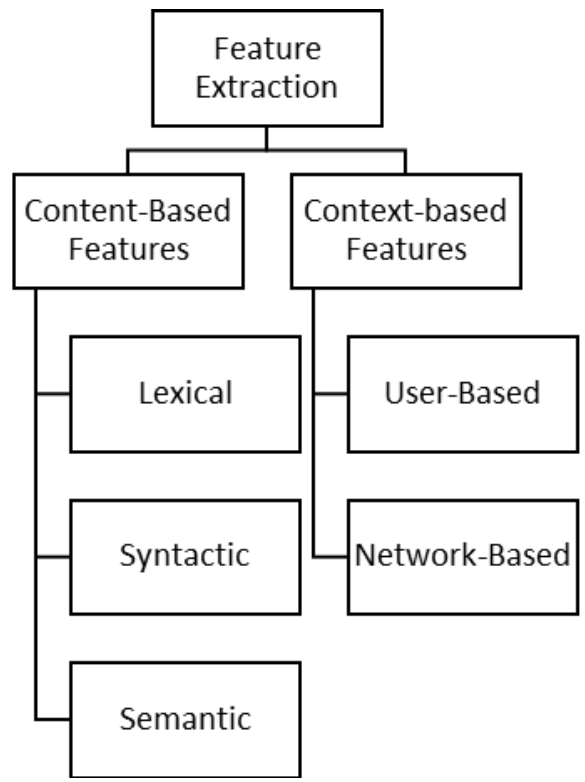

Fig. 2. Different types of features used

Clearly, the content plays a key aspect in discovering potential deception. However, using only textual features to detect false information may be inefficient to generalize in a real-world application system. Moreover, they may inefficient in descriptiveness as fake news are becoming indistinguishable in respect of writing manner with proper news. Finally, other characteristics may prove to be more effective when we are concerning only on rumors on social media.

\section{B. Context features}

The most widely used context features concern the analytics of users, sources of the rumor or news, propagation structures of the information on social media, and response of other users with respect to the news.

User-based features model characteristics of social media users (e.g., number of posts, age of the account, number of friends/followers, etc.) who create or share the news. Earliest studies on user credibleness [27] have adopted some features straightaway extracted from information on the users in Twitter, obtaining promising results. In most social media platforms, it is possible to obtain a verified status for the account. To avoid the issue of fake profiles public figures uses it very often. Clearly, it can also be efficient for the reliableness of the shared information. Other information concerns the measure of social circles and activity within the social media. This can be measured for example as the number of posts as well as by follower to following ratio.

Obviously, when considering user-based features, an essential aspect to be taken into account is approach-ability. In reality, most researches show comparable trends for what concerns such features. That is especially due to the reality that data on the users and user interactions on the platform isn't always generally open, due to privacy constraints. In addition, only a few of the information is represented in the identical manner over different social media systems.

Network-based features are used to model facts regarding the characteristics of the network in which the information is shared. For instance, propagation structures, diffusion styles, and properties of the sub-graph wherein the news is spread, which include density and clustering coefficient, are taken into consideration.

\section{APPROACHES TO DETECT FALSE INFORMATION}

Most methods aimed toward detecting fake information have focused on the use of content material features for classification. According to [24], Only a few methods for fake news detection have trusted social-context models.

Moreover, frequently researchers are forced to test distinctive type algorithms with the intention to locate the most appropriate dataset for the project. The classification approaches can be divided into techniques based on machine learning and deep learning. The subsequent subsections will talk in detail techniques based totally on machine learning and deep learning, and other procedures.

\section{A. Detection approaches based on machine learning}

Machine learning algorithms have proven to be extremely beneficial for various tasks in engineering area like image recognition, predictions, classification, etc. Because the earliest procedures specializing in credibility on social media and deception detection in computer-mediated communication had provided promising outcomes, machine learning strategies have been implemented in some of the researches concerning the trouble of fake information detection on the internet. especially, maximum machine learning procedures applied for fake information and rumor detection have employed a supervised learning approach.

\footnotetext{
${ }_{5}^{5}$ https://stanfordnlp.github.io/CoreNLP/

${ }^{\overline{6}}$ https://www.nltk.org
} 
Support Vector Machines (SVMs) are discriminative classifiers formally described by an isolating hyperplane. Experiments in [4], SVMs have obtained an F-measure F 1 of 0.84 and outperformed some of supervised machine learning techniques in textual content. however, as mentioned by the authors themselves, there exists an enormous version in performance depending on the dataset decided on for training [4].

Content material-based functions (e.g. linguistic and visual features) were exploited in maximum SVM-based techniques to fake information and deception detection $[20,4]$. Rubin et al. [20] used SVM which employed content-based features, for satirical fake news detection and obtained an F 1 of 0.87 . As regards rumor related tasks (i.e. detection, verification, and many others.), SVM-based techniques have made greater outstanding use of context-based features as well as content materialbased of content- and context-based features to train the classifiers, that are used to tag either a single social media put up or an event defined in a chain of posts as rumors or not [19]. Yu et al. [19] have proposed an extension of the feature set provided in [3]. in addition, Qin et al. [19] have additionally tweets, that has allowed reaching an accuracy of 0.75 . employed in [13], wherein the authors have proposed using a propagation systems and content features. An accuracy of 0.91 was reported on a small Sina Weibo dataset. In [23], authors have proposed a set of features to differentiate amongst fake news, actual information and satire. The titles are vital in distinguishing among actual and fake news, and that content of fake news is more similar to satire.

Using The decision tree one can determine the class by recursively splitting characteristic values. selection trees are generated from records with algorithms such as J48 (C4. five). In spite of their relative simplicity with respect to different machine learning schemes, they exhibit aggressive overall performance on the task at hand. The effectiveness of the J48 decision tree with respect to other algorithms, which includes SVM, has been proven in [3,16]. More particularly, in [3] the authors have used a combination of content-based and context-based capabilities to carry out credibility assessment of tweets, accomplishing an accuracy of 0.86 . Giasemidis et al. [16] have used a decision tree for veracity classification of rumors. The technique is capable of attaining 0.96 accuracies on the proposed dataset, with a small range of features.

Random forests [23] had been employed in several works on rumor evaluation. A random forest is an ensemble of multiple decision trees. The mode of all the predictions from each tree is the very last output. Comparative research on different machine learning algorithms for fake information detection tasks have mentioned random forests as a strong performer [16]. more particularly, Kwon et al.

Specifically, for tasks like rumor stance classification, learning algorithms based on logistic regression has been used. As for choice trees and random forests, research evaluating different techniques in the context of rumors and fake information has suggested aggressive performances for logistic regression [16]. totally ones. maximum processes have exploited a mixture provided a set of features aimed toward detecting novelty in

Another exciting SVM-based totally strategy has been graph-kernel-based SVM classifier to discover rumors using experimental outcomes mentioned in the paper propose that

logistic regression considering only headlines and claims for stance classification of news articles is used in [15] and obtained an accuracy of 0.73 on the Emergent dataset [15]. Ultimately, a method based on an ensemble of classifiers can be carried out. Ensembles depend upon the predictions of a number of unbiased classifiers and provide the very last type via a majority vote scheme. Wang [26] has trained models using several learning algorithms which used a series of context and content-based features.

\section{B. Detections approaches based on deep learning}

Deep learning classifiers have seen a remarkable rise in reputation in recent years, modernday extremely promising outcomes in trendy research fields, including textual content mining and NLP. The feature extraction project is time-consuming and can bring about biased features [17]. that is a vital difficulty for tasks consisting of fake news and rumor detection, where the identification state relevant features for the analysis may also pose a fair more challenge. alternatively, deep learning frameworks can analyze hidden representations from less difficult inputs both in context and content variations [17]. The trouble is consequently shifted from modeling relevant input features to modeling the network itself in a manner that enables the task to be solved effectively.

The 2 most broadly applied paradigms in present-day artificial neural networks are Recurrent Neural Networks (RNN) and Convolutional Neural Networks (CNN).

RNNs are a class state neural networks in which nodes are linked sequentially to one another to shape a directed graph. This structure makes RNNs, and mainly long short-term memory (LSTM) networks, especially powerful for modeling sequential facts, consisting of for example the human language, and capturing relevant features from distinctive sources state information. In the context state fake news and rumors, the one of the first use of RNNs for rumor detection is mentioned in [17]. Authors have proposed their RNN architectures, namely tanh-RNN, LSTM and Gated Recurrent Unit (GRU). Most of the proposed architectures, GRU has received high-quality outcomes in both the datasets taken into consideration, with 0.88 and 0.91 accuracies, respectively. In addition, almost all deep learning architectures had been shown to outperform traditional machine learning algorithms in terms of efficiency. The LSTM is fed with temporal information regarding the engagement state a news, user residences and textual content characteristics. The approach has been tested on the datasets proposed in [17], acquiring an accuracy of 0.89 and 0.95 , respectively. The authors in [28] have proposed a multi-task learning technique to resolve the problem of rumor classification. More particularly, they have proposed a design with multitask learning framework using an LSTM layer shared among all tasks, along with task particular layers. the performance had been evaluated at the PHEME [21] datasets. The authors obtained accuracy starting from 0.36 to 0.64 .

CNN's are a class of neural networks with an input layer, an output layer, and a sequence of hidden layers, wherein trendy transformations are applied to the facts through pooling and convolution operations.

Published By: 
Considering rumors and fake information, a number of latest works encompass CNNs. Chen et al. [22] have proposed a CNN with single and multi-phase embeddings for solving both stance and veracity classification of tweets. The authors have reported the accuracy of 0.70 for the assignment of stance classification.

Similarly, a number of latest works have exploited an aggregate of RNNs and CNNs in their models [3,25,26]. Ajao et al. [27] have performed a few experiments using LSTM and hybrid LSTM-CNN architectures. The excellent outcomes had been obtained by the most effective LSTM model, with an accuracy of modern-day 0.82 on the PHEME dataset [21]. but, the authors themselves have said that the hybrid model is expected to attain higher results given a bigger training set. Song et al. [25] have proposed to detect false rumors on social media based on repost sequence patterns. More particularly, feature vectors from posts and reposts were obtained using an CNN model, and used them as inputs to an RNN. further, authors have attempted to carry out detection as early as viable within the series of reposts primarily based on a threshold method. The approach has been tested on the datasets in [17], obtaining accuracies above 0.90 .

\section{Other approaches}

Apart from machine and deep learning-based classification approaches, different studies have attempted with some of specific approaches for detection of fake news and rumors. The importance of such processes is twofold. They can constitute precious alternatives to machine learning and, more in general, classification approaches, by probably exploiting unique characteristics of false information. The approaches discussed in the following uses different from those presented above.

A framework for evaluating tweets and their authors as possible sources of incorrect information has been proposed in [7]. Specifically, the credibility of a tweet became first evaluated by using measuring disparity in retweet conduct through the Gini coefficient. Credible tweets along with their retweets are then used to build a retweet graph. The PageRank algorithm [1] changed into then used to compute a score of acceptability for the given tweet. curiously, such statistics is then provided to customers with the objective of permitting them to carry out a more knowledgeable assessment of the tweet.

An variation of the algorithm proposed in [10] has been used which will analyze Facebook users generally tend to like either hoaxes or non-hoaxes. The version has been then tested at the proposed dataset, obtaining 0.99 accuracies.

Finally, techniques based on computational-orientated fact-checking [24] are well worth to be referred to. despite the fact that the task of fact-checking can be taken into consideration as slightly distinctive from fake news and rumor detection, it is however absolutely comparable to them. the primary attempt is addressed to carry out automatically fact-checking. some of techniques were proposed to this intention. Wu et al. [] $]$ has delivered the task of automated fact-checking and offered a series of algorithms for solving the assignment via automatically designing queries aimed at checking whether or not the statement is true or false. Another widely used technique uses knowledge graphs. For example, it is employed in research [9]. More particularly, the usage of models, such as clustering and vector space models,

Wikipedia info-boxes to generate a information graph has been proposed in [9]. here, the authors have described a measure of semantic proximity via the use of a transitive closure set of rules so as to check claims towards the knowledge graph.

In our evaluation of the different works provided above, we say that different strategies have been proposed in various literature, exploiting various techniques. The performances, expressed in terms of accuracy or F-measure, of those techniques are pretty different. But, we cannot compare different techniques and evaluate whether one technique is more performing than another as they work on different dataset and their does not exist an standard dataset. despite the fact that, we've got determined to report the overall performance offered in the papers to allow the reader to have a glimpse of what they could anticipate at the state of the art in terms of performance for this type of application area.

\section{FUTURE DIRECTIONS}

The problems discussed in this paper are truly relevant, from both real-world and research standpoints. The subjects of fake news and rumors on the internet are quite new. but, our evaluation of the literature has shown that they're a thriving area of studies and outcomes received from both the studies and application perspectives are indeed very promising. We anticipate in the truth that previously discussed methods and techniques could be in addition stepped forward and will have the capacity to be carried out into actual-global applications to combat the unfold of rumors and fake information online. but, it's far clear that development remains wished regarding many components of the discussed problems.

We will now, in brief, discuss trendy the most important challenges in fake news and rumors detection and will offer perception on viable future guidelines for studies.

First and most important, the shortage of broadly accepted benchmark datasets, particularly concerning fake information and related social media posts, needs to be addressed. that is essential to evaluate the effectiveness of the technique and examine the strategies amongst them. In truth, available resources might not be sufficient for: (i) gaining novel insight on applicable properties of fake information and rumors and (ii) constructing models able to properly function in an actual world scenario. In Zubiaga et al. [21], concerning social media posts and discussions in the course of breaking news events that have accumulated real and fake statements from information and social media with the number of other context-based features. research on collecting information for this sort of analysis must focus on constructing large scale datasets and most significantly at the identification of a clean and popular benchmark for evaluation. large scale datasets should allow analysis on a stage more similar to actual situations. moreover, given the trouble of clearly figuring out applicable functions for the analysis, as tons data as feasible ought to be accumulated so as to gain deeper insight at the problem.

Our assessment of the literature has proven a clean trend in want of supervised classification procedures to fake information and rumors detection. 
The trend will likely retain in future years, as supervised classification models based on deep learning keep growing in popularity among researchers for a huge variety of tasks. In reality, deep learning techniques have acquired state-of-the-art outcomes, and the latest tactics are targeted on exploiting such frameworks to a degree.

This might be cutting-edge the truth that, not like conventional machine learning approaches, no attempt required by feature engineering is wanted to gain a reliable prediction. feature engineering is in truth an area in which we are able to identify substantial room for improvement, for two most important reasons. First, fake news and rumors nonetheless need to be fully understood from a linguistic attitude [24], and research around telltale linguistic-based capabilities has the capacity of creating numerous steps forward. second, given the streaming nature latest social flow. this means that the relevance of information, as well as for a given event or time frame, may not scale and generalize well for actual-global applications or information from a distinctive context. For these motives, future studies on this direction ought to focus on higher knowledge of the significance of certain capabilities for classification, in possibly manage concept drift in a real-world situation. furthermore, the use of visual features has no longer obtained much attention within the literature. however, as image and video manipulation gear come to be available to wider audiences, visual features to differentiate between authentic and fake content material are increasingly essential

Given our evaluation of the available literature, we will argue that the best procedures implement each content-based and context-based feature to construct a classifier with higher efficiency. This has ended up apparent especially in rumor related studies, in which we can sincerely discover a trend to use as much information as possible for detection. Hybrid methods, that may simultaneously model-specific elements of fake news, together with the real text, diffusion patterns and stance of the news, no matter is more complicated in terms of models, information availability and a number of features may be suited to better solve the problem and will be absolutely pursued.

Finally, semi-supervised or unsupervised models may be explored as a feasible solution for further research work. The primary benefit of exploiting such strategies is given by the fact that they are able to learn from unlabeled information, as a result mitigating the trouble of locating and labeling relevant statistics. furthermore, such fashions can also permit a higher understanding of the problem and of its key traits.

\section{CONCLUSION}

In this paper, we explored topic of potentially false information and discussed various efforts made in past for its detection and evaluated this efforts by considering the accuracy they obtained on their dataset.

Fake information and rumors have come to be a vital aspect of our digital lives. they have already proven to be media, records will be a problem to concept go with the proposed capabilities, could vary through the years. both content material based and context-based features, extracted addition to their capacity to generalize at the problem and and could be studied more in-depth.

potentially very dangerous in the digital environment as well as outside of it.

In this paper, we've provided an outline of the current state of the art techniques and methods to the problem of detecting such fake information on the internet. Our assessment has especially focused on 5 key elements. First, we've provided clear definitions and distinctions for the different sub-issues such as fake information and rumors. studies on such subjects has in reality produced several different, albeit similar, definitions. we have attempted to make the needed clarity in the debate on fake information and rumors. second, we've got highlighted valuable sources of relevant information and techniques to acquire them. for the reason, that field is pretty novel, no widely familiar method for retrieving and labeling information has been proposed. accordingly, numerous researchers face the same problem with barely different goals. despite the fact that a significant attempt has been undertaken within the direction modern publicly to be had resources, a extensively general benchmark dataset has no longer emerged yet. third, we have focused on the different elements of fake information and rumors that may be implemented for detection, along with content-based and context-based features. Fourth, we have supplied an overview of techniques to perform fake news and rumor detection and classification. Our assessment highlights how the main studies fashion is that brand new imposing supervised gadget state-of-the-art strategies. More particularly, researcher's focus from machine learning algorithms to deep learning algorithms have been noticed recently for the classification purpose. Deep learning techniques have received stateof-the-art accuracy in most, if not all, instances. in addition, we've provided a description of the most promising alternative techniques, that either use unsupervised or semi-supervised learning algorithms or enforce absolutely different techniques to carry out the evaluation.

In the end, we have provided some insight into feasible future trends for this area of study. Such traits encompass enhancement of existing techniques by identifying novel relevant features for analysis in addition to the identification of alternative strategies that could better perform in a real-world scenario.

Subjects presented in this review are expected to turn out to be prominent in the discussion around social media, both from a social and a research standpoint. trendy, we believe that further research on this course is needed, as such contributions will truly play a vital role in shaping the future of online conversation.

\section{REFERENCES}

1. O. Ajao , D. Bhowmik, S. Zargari, Fake news identification on twitter with hybrid CNN and RNN models, in: Proceedings of the Ninth International Conference on Social Media and Society, ACM, New York, NY, USA, 2018, pp. 226-230 .

2. C. Castillo, M. Mendoza , B. Poblete , Information credibility on twitter, in: Proceedings of the Twentieth International Conference on World Wide Web, ACM, Hyderabad, India, 2011, pp. 675-684 .

3. Y.-C. Chen , Z.-Y. Liu , H.-Y. Kao , Ikm at semeval-2017 task 8: convolutional neural networks for stance detection and rumor verification, in: Proceedings of the Eleventh International Workshop on Semantic Evaluation (SemEval-2017), 2017, pp. 465-469 . 
4. G.L. Ciampaglia , P. Shiralkar, L.M. Rocha , J. Bollen , F. Menczer , A. Flammini , Computational fact checking from knowledge networks, PloS One 10 (6) (2015) .

5. L. De Alfaro , V. Polychronopoulos , M. Shavlovsky, Reliable aggregation of boolean crowdsourced tasks, in: Proceedings of the Third AAAI Conference on Human Computation and Crowdsourcing, 2015, pp. 42-51

6. L. Derczynski , K. Bontcheva , Pheme: veracity in digital social networks, in: Proceedings of the Tenth Joint ACL ISO Workshop on Interoperable Se- mantic Annotation (ISA), Reykjavik, Iceland, 2014, pp. 19-22

7. N. Di Fonzo, P. Bordia, Rumor, gossip and urban legends, Diogenes 54 (1) (2007) 19-35

8. W. Ferreira, A. Vlachos, Emergent: a novel data-set for stance classification, in: Proceedings of the Conference of the North American Chapter of the Association for Computational Linguistics: Human Language Technologies, 2016, pp. 1163-1168.

9. G. Giasemidis , C. Singleton , I. Agrafiotis , J.R. Nurse , A. Pilgrim , C. Willis , D.V. Greetham, Determining the veracity of rumours on twitter, in: Proceedings of the International Conference on Social Informatics, Springer, 2016, pp. 185-205 .

10. B.D. Horne, S. Adali, This just in: fake news packs a lot in title, uses simpler, repetitive content in text body, more similar to satire than real news, 2017 [Online Available: https://arxiv.org/abs/1703.09398 ]

11. E. Kochkina , M. Liakata , A. Zubiaga , All-in-one: multi-task learning for rumour verification, in: Proceedings of the Twenty Seventh International Conference on Computational Linguistics, 2018, pp. 3402-3413

12. K.P.K. Kumar, G. Geethakumari, Detecting misinformation in online social networks using cognitive psychology, Human-centric Comput. Inf. Sci. 4 (1) (2014) 14

13. J. Ma , W. Gao, P. Mitra , S. Kwon, B.J. Jansen , K.-F. Wong, M. Cha , Detecting rumors from microblogs with recurrent neural networks, in: Proceedings of the Twenty-Fifth International Joint Conference on Artificial Intelligence, IJCAI, address, 2016, pp. 3818-3824

14. T. Mikolov, I. Sutskever, K. Chen, G. Corrado, J. Dean, Distributed representations of words and phrases and their compositionality, in: Proceedings of the Twenty Sixth International Conference on Neural Information Processing Systems - Volume 2, in: NIPS, Curran Associates Inc., USA, 2013, pp. 3111-3119.

15. T. Mitra, E. Gilbert, CREDBANK: a large-scale social media corpus with associated credibility annotations, in: Proceedings of the Ninth International AAAI Conference on Web and Social Media, 2015, pp. 258-267.

16. L. Page, S. Brin, R. Motwani, T. Winograd, The PageRank citation ranking: bringing order to the web, Technical Report, Stanford InfoLab, 1999

17. M. Potthast, S. Köpsel, B. Stein , M. Hagen, Clickbait detection, in: Proceedings of the European Conference on Information Retrieval, Springer, 2016, pp. 810-817.

18. Y. Qin, D. Wurzer, V. Lavrenko, C. Tang, Spotting rumors via novelty detection, CoRR (2016) . [Onilne. Available: https://arxiv.org/abs/1611.06322 ]. [77] J.R. Quinlan , C4.5: Programs for Machine Learning, Morgan Kaufmann Publishers Inc., San Francisco, CA, USA, 1993.

19. V. Rubin , N. Conroy, Y. Chen , S. Cornwell, Fake news or truth? Using satirical cues to detect potentially misleading news, in: Proceedings of the NAACL-CADD Second Workshop on Computational Approaches to Deception Detection, San Diego, California, USA, 2016, pp. 7-17

20. V.L. Rubin , Y. Chen , N.J. Conroy, Deception detection for news: three types of fakes 52 (1) (2015) 1-4.

21. K. Shu, A. Sliva, S. Wang, J. Tang, H. Liu, Fake news detection on social media: a data mining perspective, ACM SIGKDD Explor. Newslett. 19 (1) (2017) 22-36 .

22. C. Song, C. Tu, C. Yang, Z. Liu, M. Sun, CED: credible early detection of social media rumors, 2018 . [Online. Available: https://arxiv.org/abs/1811.04175 ]. [88] E. Tacchini, G. Ballarin, M.L. Della Vedova, S. Moret, L. de Alfaro, Some like it hoax: automated fake news detection in social networks, CoRR (2017). [Online. Available: https://arxiv.org/abs/1704.07506 ].
23. W.Y. Wang, "Liar, liar pants on fire": a new benchmark dataset for fake news detection, in: Proceedings of the Fifty Fifth Annual Meeting of the Association for Computational Linguistics (Volume 2: Short Papers), Association for Computational Linguistics, 2017, pp 422-426

24. K. Wu, S. Yang, K.Q. Zhu, False rumors detection on Sina Weibo by propagation structures, in: Proceedings of the IEEE Thirty First International Conference on Data Engineering (ICDE), IEEE, 2015, pp. 651-662

25. Y. Wu , P.K. Agarwal, C. Li , J. Yang, C. Yu, Toward computational fact-checking, Proc. VLDB Endow. (PVLDB) 7 (7) (2014) 589-600

26. H. Zhang, Z. Fan, J.-h. Zheng, Q. Liu , An improving deception detection method in computer-mediated communication, J. Netw. 7 (11) (2012) 1811-1816

27. A . Zubiaga , A . Aker, K. Bontcheva , M. Liakata , R. Procter, Detection and resolution of rumours in social media: a survey, ACM Comput. Surv. 51 (2) (2018) 32:1-32:36 .

28. A. Zubiaga , M. Liakata , R. Procter , K. Bontcheva , P. Tolmie , Towards detecting rumours in social media, in: Proceedings of the AAAI Workshop: AI for Cities, 2015, pp. 35-41

29. A. Zubiaga , M. Liakata , R. Procter , G. Wong Sak Hoi , P. Tolmie , Analysing how people orient to and spread rumours in social media by looking at conversational threads, PLOS ONE 11 (3) (2016) 1-29.

\section{AUTHORS PROFILE}

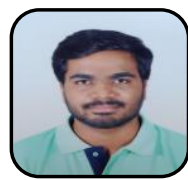

\section{Henil Chopada}

I am a computer engineering undergraduate student at Babaria Institute of Technology. I have programming skills and have worked on several projects on different platforms. I have also won several competitive programming competitions. Currently, I am working on "Fake News Detection using ML/NLP" as my final year undergraduate project.

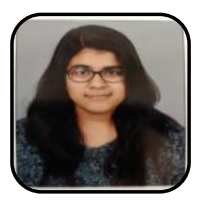

\section{Maitri Patel}

I am an undergraduate student at Babaria Institute of Technology, Vadodara. My areas of interest include distributed systems, software testing and quality assurance. Throughout these 4 years of my undergraduate studies, I have worked on several projects based on different coding platforms. I am currently working on the project based on fake news detection using Machine Learning and natural language processing.

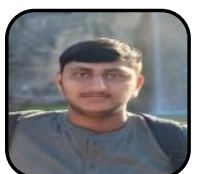

Rushikesh Desai I Rushikesh Desai a student of Computer Science and engineering, doing my Bachelor from Babaria Institute of technology. I developed interest in this field and started off learning $\mathrm{C} / \mathrm{C}++$ and basics of computer science on Coursera and Udemy. Nowadays I am working on small projects to gain more experience in coding. However, I am familiar with the other programming language such as java, python, HTML, and XML. cybersecurity is also my part of interest.

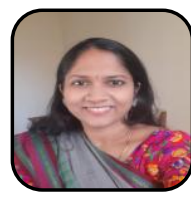

Mrs. Divya Ebenezer Nathaniel

I have been working as assistant professor at Babaria Institute of Technology since 2006. I have teaching experience of 15 years and I have guided several under graduate projects and her area of interest include image processing, distributed systems, machine learning she acquired my MTech from Anna university Chennai. 